\title{
In Situ Determination of Density Profiles in Complex Strata Using the Nuclear Density Cone Penetrometer
}

\author{
Rui Jia $\mathbb{D}^{1,2}$ Huayang Lei $\mathbb{D}^{1,2}$ Wenjun Zhang, ${ }^{1,2}$ and Haizuo Zhou $\mathbb{C}^{1,2}$ \\ ${ }^{1}$ School of Civil Engineering, Tianjin University, 135 Yaguan Road, Jinnan District, Tianjin 300350, China \\ ${ }^{2}$ Key Laboratory of Coast Civil Structure Safety of Ministry of Education, Tianjin University, 135 Yaguan Road, Jinnan District, \\ Tianjin 300350, China
}

Correspondence should be addressed to Huayang Lei; leihuayang74@163.com

Received 7 February 2019; Accepted 28 May 2019; Published 18 June 2019

Academic Editor: Roberto Palma

Copyright (c) 2019 Rui Jia et al. This is an open access article distributed under the Creative Commons Attribution License, which permits unrestricted use, distribution, and reproduction in any medium, provided the original work is properly cited.

\begin{abstract}
The nuclear density cone penetrometer (ND-CP) is an in situ testing device that can provide continuous data on soil density, but it measures the composite density $\left(\rho_{\mathrm{c}}\right)$ of the soil within a spheroid centered at the midpoint between the gamma ray source and the detector. A theoretical model for predicting $\rho_{\mathrm{c}}$ of the ND-CP is proposed, and equations for calculating $\rho_{\mathrm{c}}$ are derived when the ND-CP penetrates into strata with different functions of density distributions. The calculated $\rho_{c}$ profiles provide a good fit to the laboratory-measured $\rho_{\mathrm{c}}$ profiles by the ND-CP, indicating that the proposed theoretical model can be used to calculate the $\rho_{\mathrm{c}}$ within the spheroid. The comparisons of the density $(\rho)$ and $\rho_{\mathrm{c}}$ values show that the $\rho_{\mathrm{c}}$ profiles are considerably different from the actual $\rho$ profiles at the boundaries of each stratum where density changes suddenly. Therefore, a method for deducing the actual $\rho$ profiles from the measured $\rho_{\mathrm{c}}$ profiles in complex strata is proposed, and this method is used to determine the actual $\rho$ profiles from the ND-CP measured $\rho_{\mathrm{c}}$ profiles in the field. The research results would be beneficial for the in situ determination of the density profile in complex strata using the ND-CP.
\end{abstract}

\section{Introduction}

Soil density is defined as the mass of the soil per unit of volume. It depends on the density of the solid constituents, the porosity of the aggregate, and the degree of saturation $[1,2]$. Soil density measurement is important because it determines the degree of compactness as a measure of soil structure and is used for calculating the pore space of soils [3]. The density of soil is an indispensable parameter used for the classification of stratigraphy, identification of soft interlayers, determination of the relative density of sand, assessment of the effect of ground improvement, and calculation of a series of soil physical indexes $[4,5]$. Furthermore, the density of bed sediments is an important parameter used for predicting sediment transport, determining nautical depth, and planning dredging projects [6].

There are mainly two types of methods available for soil density measurement. One is the long-established direct method, which involves the measurement of the sample mass and volume. The other is the indirect nondestructive method, which involves measuring the attenuation or scattering of gamma radiation by the soil and determining the soil density from the intensities of the original and the attenuated gamma radiation. The nondestructive method is equal in accuracy to the direct method of density determination and is simpler and quicker to use, especially where measurements at different depths are required [7]. It can be classified into the backscatter type and direct transmission type. In backscatter gauges, the gamma radiation source and detector are leadshielded and located on the soil surface or are lowered into an access hole in the soil. The gamma rays emitted from the source enter into and interact with the soil and attenuate by scattering. In this process, some of the rays that are scattered towards the detector are counted. In transmission gauges, the gamma radiation source emits rays that enter into and interact with the soil, and some of the rays are scattered away from the detector. The rays passing through the soil are detected and counted by the detector [8]. 
The use of gamma radiation to measure density is a geophysical technique that was first applied in oil well logging [9]. Subsequently, this technique has been used for a number of years in geotechnical engineering to measure the wet density of soils [10-14]. With recent advances in electronics and the miniaturization of various electronic sensors and devices, the original bulky nuclear density gauge has been developed and incorporated into the piezocone penetrometer, which is the most versatile tool currently available for in situ soil investigation $[15,16]$; thus, the nuclear density cone penetrometer (ND-CP) was developed [17-19]. The ND-CP has been used in soil investigations, and it can provide reliable soil density measurements [20-23].

In the ND-CP, the density is calculated by the count rate ratio $\left(R_{\rho}\right)$ of the gamma rays detected by the detector to the gamma rays emitted from the source. Thus, the NDCP measures the composite density $\left(\rho_{\mathrm{c}}\right)$ of the soil within a spheroid centered at the midpoint between the gamma ray source and the detector [24]. Therefore, the measured $\rho_{\mathrm{c}}$ is not the actual $\rho$ of the soil at a certain depth in the ground. There may be a considerable difference between the measured $\rho_{\mathrm{c}}$ profile and the actual $\rho$ profile at the boundaries of each stratum, where the density changes suddenly, e.g., in layered strata, strata with soft interlayers, and bed sediments. Therefore, it is necessary to study how to determine the actual $\rho$ profiles in complex strata from the measured $\rho_{\mathrm{c}}$ profiles by the ND-CP.

In this study, the density measurement principle and the density measurement volume of the ND-CP are described first. Then, a theoretical model for predicting $\rho_{\mathrm{c}}$ is proposed when the ND-CP penetrates into strata with different density distribution functions. The theoretical model is verified by comparing the calculated $\rho_{\mathrm{c}}$ profiles by the theoretical model with the measured $\rho_{\mathrm{c}}$ profiles by the ND-CP in the laboratory. Then, a method for deducing the actual $\rho$ profile from the measured $\rho_{\mathrm{c}}$ profile is proposed based on the difference between the $\rho$ profiles and the $\rho_{\mathrm{c}}$ profiles in complex strata. Finally, the proposed method is used to determine the actual $\rho$ profiles from ND-CP measured $\rho_{\mathrm{c}}$ profiles in the field.

\section{Measurement Principle of Nuclear Density Cone Penetrometer}

2.1. Nuclear Density Cone Penetrometer. Figure 1 shows the ND-CP. The ND-CP mainly consists of a standard piezocone penetrometer and a nuclear densitometer. The piezocone penetrometer is identical to the standard electronic cone. The nuclear densitometer mainly consists of a gamma ray source and a gamma ray detector. The diameters of the piezocone penetrometer and the nuclear densitometer are $35.7 \mathrm{~mm}$ and $48.6 \mathrm{~mm}$, respectively. The distances of the cone resistance sensor, the pore pressure sensor, the sleeve friction sensor, the inclinometer sensor, the gamma ray source, the density count gamma ray detector, and the background count gamma ray detector from the apex of the cone are $0 \mathrm{~m}, 0.04 \mathrm{~m}$, $0.11 \mathrm{~m}, 0.5 \mathrm{~m}, 0.721 \mathrm{~m}, 0.986 \mathrm{~m}$, and $1.4535 \mathrm{~m}$, respectively [24].
2.2. Density Measurement Principle. Gamma rays are a byproduct of the natural decay of some radionuclides. The density measurement principle of the ND-CP is based on the absorption of gamma rays (interaction between the rays and the soil that reduces the intensity of the rays). The photons of the gamma rays can interact with the electrons of the soil. Three different interaction processes, namely, the photoelectric effect, Compton scattering, and pair production, are observed. The photoelectric effect is the interaction of a gamma photon with an electron in the inner shell of the atom. The gamma photon is completely absorbed, and the electron uses the additional energy to leave its atom as a free electron. The Compton effect is the scattering of a gamma photon by an inner shell electron, whereby only part of the photon energy is transferred to the electron. The energy and direction of the gamma photon thus change, and the electron leaves its electronic shell. Pair production is a process in which a gamma ray of sufficient energy is converted into an electron and a positron. Energies of gamma rays range from approximately $10 \mathrm{keV}$ to $10 \mathrm{MeV}$. The photoelectric effect generally predominates at lower ray energies, the Compton effect predominates when the energy of the ray ranges from 1 $\mathrm{MeV}$ to $5 \mathrm{MeV}$, and pair production occurs at ray energies of several MeVs [25].

The principle of the soil density measurement in the ND$\mathrm{CP}$ utilizes the Compton scattering of gamma rays. When the ND-CP penetrates the ground, the gamma rays emitted from the source pass through the soil particles in the ground, interact with the $\mathrm{SiO}_{2}$ in the soil particles, collide with the electrons in the outer shell, and scatter repeatedly with the atomic electrons in the soil (Figure 2). In this process, some of the gamma rays are absorbed by the soil, while others reach the detector. The absorption depends on the density of the soil. The higher the density is, the more the electrons are available within a given volume to interact with the gamma rays, and the more gamma rays are absorbed. Hence, the soil density can be determined from the intensities of gamma rays emitted from the source and detected by the detector.

The count rate cannot be directly used to calculate density because the radioactive materials decay exponentially with time. The decay rate is characterized by the half-life of a radioisotope, which is defined as the time taken for half the original number of radioactive nuclei to decay. The gamma ray source used in the $\mathrm{ND}-\mathrm{CP}$ is the radioactive isotope cesium-137, which has a half-life of approximately 30 years. Therefore, the measured count rate must be normalized by the standard count rate to account for the radioactive decay. In addition, to obtain the accurate absorption of gamma radiation in the soil, the amount of natural gamma rays in the ground must be measured and subtracted from the total count rate detected by the detector. The count rate ratio $\left(R_{\rho}\right)$, which is used to calculate the soil density, is defined as follows [24]:

$$
R_{\rho}=\frac{D C R-B C R}{S C R}
$$

where $D C R$ is the density count rate, which is measured by the density count gamma ray detector; $B C R$ is the background count rate, which is measured by the background count 


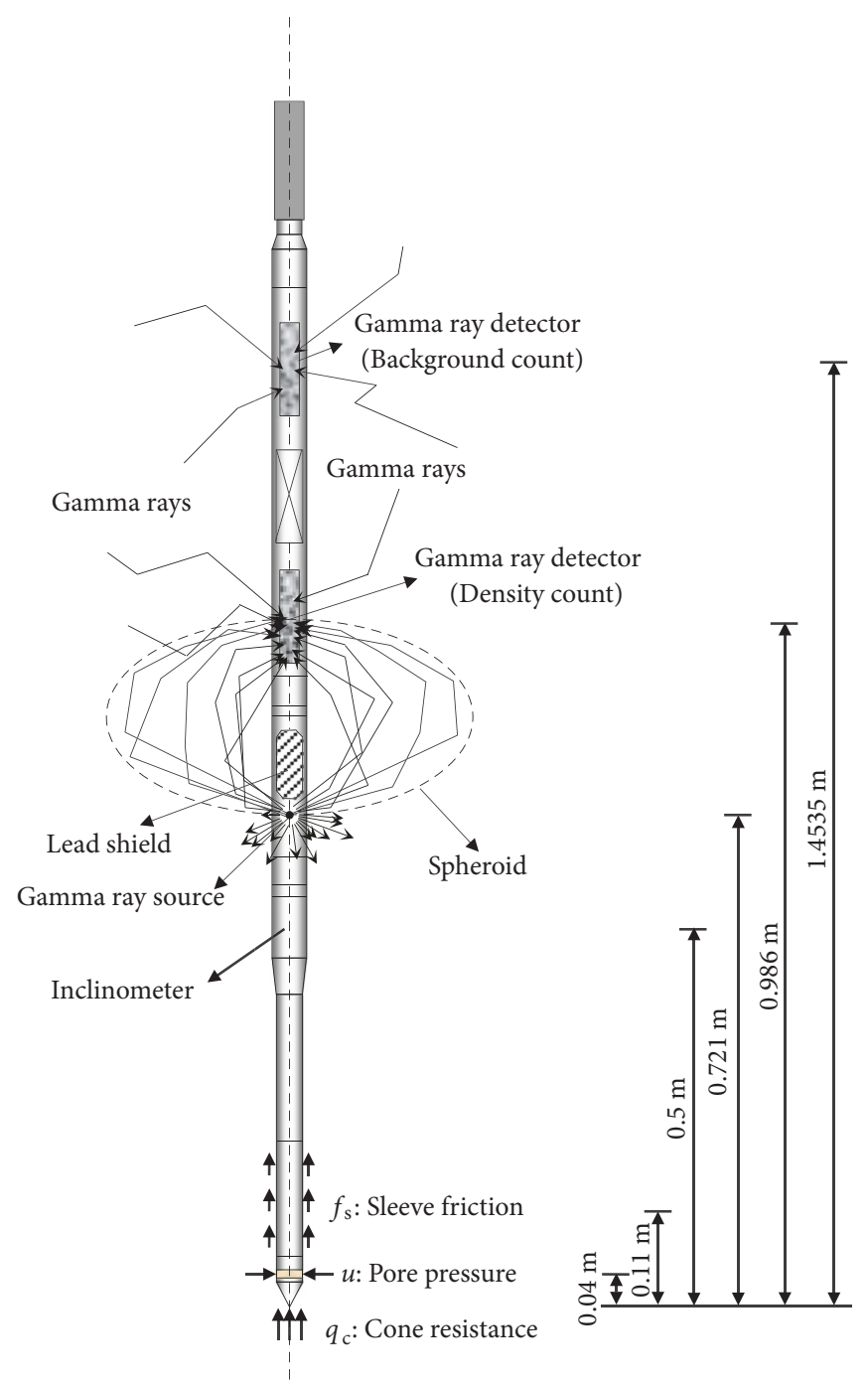

FIGURE 1: Nuclear density cone penetrometer.

gamma ray detector; and $S C R$ is the standard count rate, which is measured in an inactive material under controlled conditions before each in situ investigation [26]. The calibration equation between soil density and $R_{\rho}$ is as follows [24]:

$$
R_{\rho}=A \rho^{2}-B \rho+C
$$

where $\rho$ is density and $A, B$, and $C$ are $0.6264,4.0954$, and 6.8422, respectively, for density values ranging from 1.0 $\mathrm{g} / \mathrm{cm}^{3}$ to $2.2 \mathrm{~g} / \mathrm{cm}^{3}$. Theoretically, the ND-CP can be used to measure the density of soil up to $2.2 \mathrm{~g} / \mathrm{cm}^{3}$. However, similar to the piezocone penetrometer, the $\mathrm{ND}-\mathrm{CP}$ is applicable to general clay, silt, and sand, especially to loose soil and mucky soil below the groundwater level, for which undisturbed samples are difficult to obtain.

2.3. Density Measurement Volume. The absorption of gamma rays and the measured $D C R$ depend on the density of the soil within a spheroid between the gamma ray source and the detector, as shown in Figure 1. The measurement volume is an oblate spheroid centered at the midpoint between the gamma ray source and detector (Figure 3 ). The length of semiaxis $b$, which is the distance from the center to the pole along the symmetry axis, is half of the gamma ray sourcedetector separation distance $(2 b)$ of the ND-CP. In this study, the value of $2 b$ is $26.5 \mathrm{~cm}$. The length of semiaxis $a$, which is the equatorial radius of the spheroid, decreases with an increasing density of the surrounding soil [10]. The lengths of $a$ are approximately $24 \mathrm{~cm}, 20 \mathrm{~cm}$, and $17 \mathrm{~cm}$ in water, clay, and sand, respectively [24]. Notably, the measured DCR is more sensitive to the density of the soil closer to the center of the spheroid.

The density measured by the ND-CP is the composite density $\left(\rho_{\mathrm{c}}\right)$ of the soil within a spheroid, as shown in Figure 3. Thus, the measured density $\left(\rho_{\mathrm{c}}\right)$ is not the actual density $(\rho)$ at a certain depth. If the density changes suddenly with depth, e.g., as in layered soils, the measured $\rho_{\text {c }}$ profile will be different from the actual $\rho$ profile. 


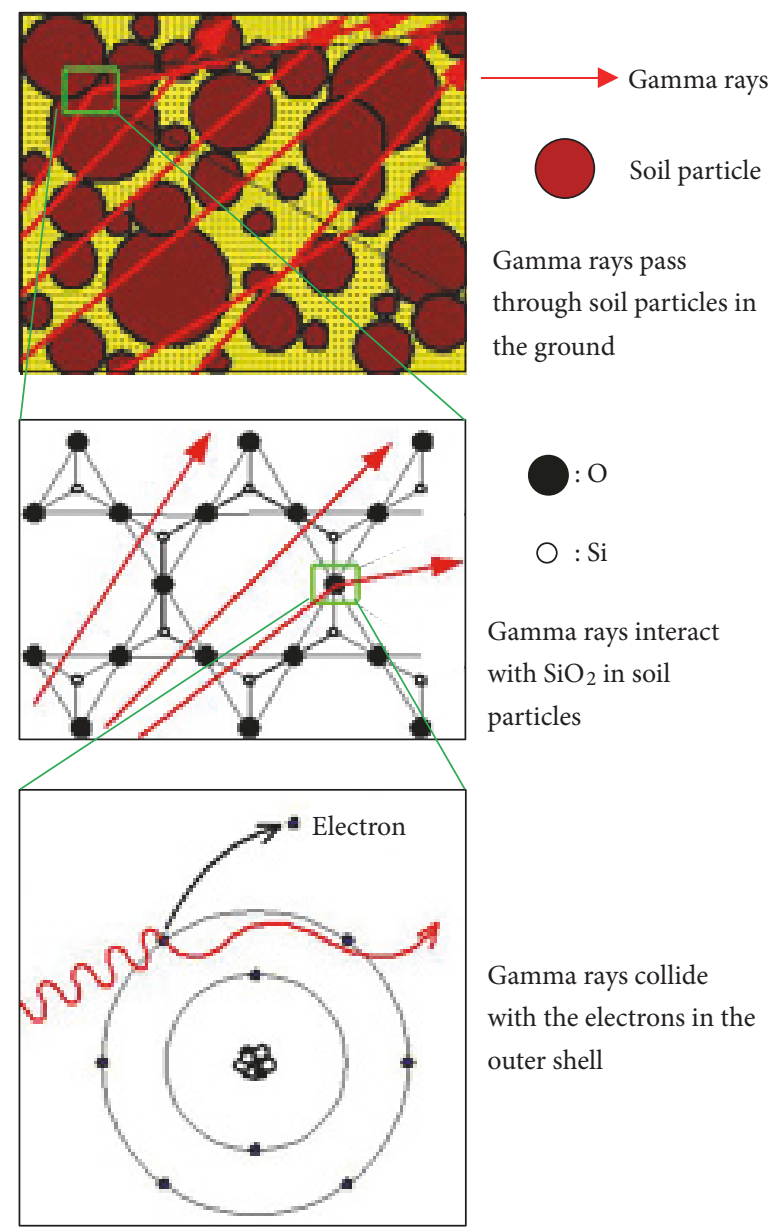

FIGURE 2: Schematic diagram of Compton scattering of gamma rays in the soil (modified from http://www.soilandrock.co.jp/).

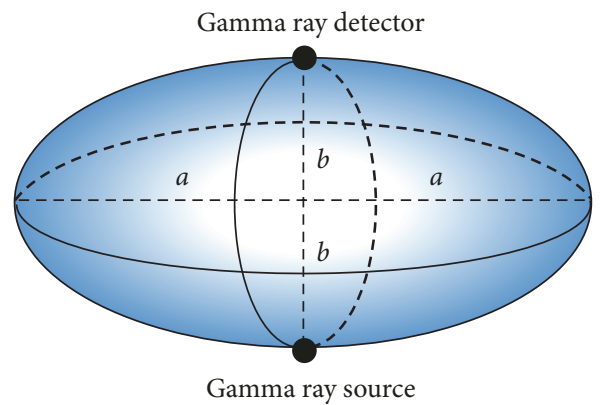

FIGURE 3: Density measurement volume of the ND-CP.

\section{Theoretical Model for Calculating Composite Density}

3.1. Theoretical Model for Predicting Count Rate Ratio. A mathematical model is proposed for the ND-CP to predict the count rate ratio $\left(R_{\rho}\right)$ when the ND-CP spans two or more strata. Then, the composite density $\left(\rho_{\mathrm{c}}\right)$ within the measurement spheroid can be calculated by (2). Figure 4 shows the spheroid coordinates for the theoretical model used to calculate $R_{\rho}$ [24]. The adopted assumptions are as follows.
(1) It is assumed that the scattering and attenuation of gamma rays occur over a spheroid volume; thus, the volume affecting the measured $R_{\rho}$ is a spheroid [27]. The center of the spheroid is at the midpoint between the source and the detector. The vertical section of the spheroid is an ellipse, and the horizontal section of the spheroid is a circle.

(2) The spheroid volume is constant. The length of semiaxis $b$ is equal to half of the gamma ray source-detector separation distance $(26.5 \mathrm{~cm})$ of the ND-CP. The length of semiaxis $a$ decreases with increasing density of the surrounding soil [10]. For simplification, it is assumed to be a constant. 


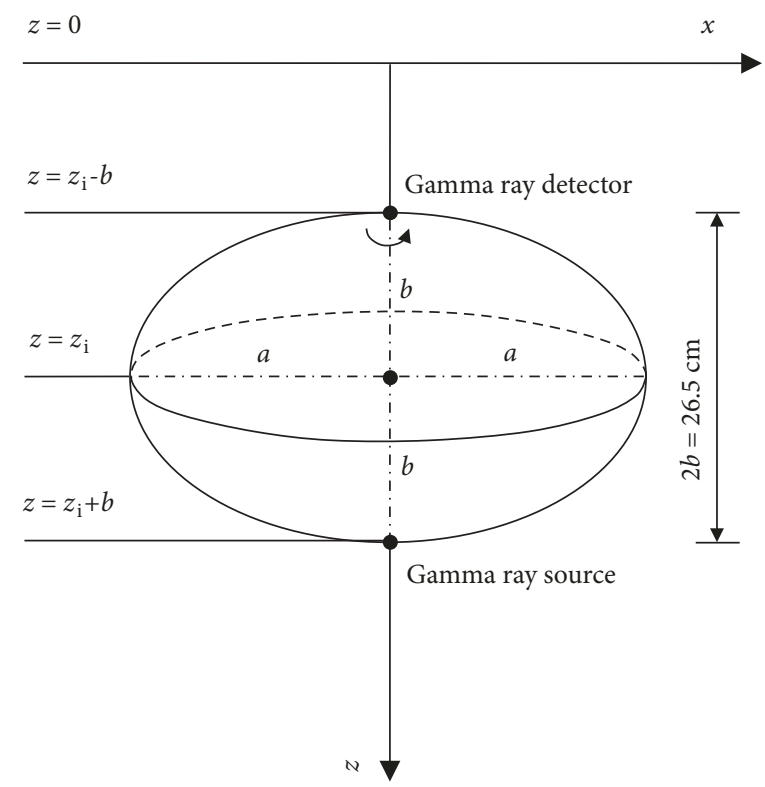

FIGURE 4: Spheroid coordinates for the theoretical model to calculate the count rate ratio.

(3) All parts of the spheroid contribute equally to the scattering and attenuation of radiation, and the contribution of the count rate ratio from a small volume $\left(\Delta R_{\rho}\right)$ in the spheroid to the total count rate ratio $\left(R_{\rho}\right)$ is proportional to its volume. This assumption is not strictly true but does not lead to a large error [28].

(4) The calibration equation between $R_{\rho}$ and density is defined by (2) for the soils with densities ranging from 1.0 $\mathrm{g} / \mathrm{cm}^{3}$ to $2.2 \mathrm{~g} / \mathrm{cm}^{3}$.

The volume of the spheroid is as follows:

$$
V=\frac{4}{3} \pi a^{2} b
$$

The vertical cross-section of the spheroid in the $x-z$ plane is an ellipse, and its equation is

$$
\frac{x^{2}}{a^{2}}+\frac{\left(z-z_{i}\right)}{b^{2}}=1
$$

where $a$ is the length of the major semiaxis, $b$ is the length of the minor semiaxis, $z$ is the depth, and $z_{\mathrm{i}}$ is the depth corresponding to the midpoint of the ellipse. The horizontal cross-section of the spheroid at any point is a circle of radius $x$, where $0<x<a$. A small volume of the spheroid $\partial V$ is

$$
\partial V=\pi x^{2} \delta z
$$

where $\delta z$ is the differential of $z$. The $x$ calculated by (4) can be substituted into (5) to give

$$
\partial V=\frac{\pi a^{2}}{b^{2}}\left[b^{2}-\left(z-z_{i}\right)^{2}\right] \delta z
$$

The assumption is that the contribution of the small volume $\partial R_{\rho}$ to $R_{\rho}$ is proportional to its volume:

$$
\frac{\partial R_{\rho}}{R_{\rho}}=\frac{\partial V}{V}
$$

Substituting (2), (3), and (6) into (7) gives

$$
\partial R_{\rho}=\frac{3\left(A \rho^{2}-B \rho+C\right)}{4 b^{3}}\left[b^{2}-\left(z-z_{i}\right)^{2}\right] \delta z
$$

Assuming the density $\rho$ is a function of depth $\rho=\rho(z)$, integrating (8) over the spheroid volume gives $R_{\rho}$ :

$$
R_{\rho}=\frac{3}{4 b^{3}} \int\left(A \rho^{2}(z)-B \rho(z)+C\right)\left[b^{2}-\left(z-z_{i}\right)^{2}\right] \delta z
$$

3.2. Equations for Calculating the Count Rate Ratio. If the density function $\rho(z)$ of the strata is known, (9) can be used to calculate the count rate ratio $\left(R_{\rho}\right)$ when the ND-CP penetrates into the strata. The probable density profiles in the ground are described by constant, linear, parabolic, and square root functions (Figure 5), the equations of which are as follows:

$$
\begin{aligned}
& \rho(z)=\rho_{0} \\
& \rho(z)=\rho_{1}+\beta_{1}\left(z-z_{1}\right) \\
& \rho(z)=\alpha_{1}\left(z-z_{1}\right)^{2}+\rho_{1} \\
& \rho(z)=\alpha_{2}\left(z-z_{1}\right)^{1 / 2}+\rho_{1}
\end{aligned}
$$

If the density and depth at the top of the stratum $\left(\rho_{1}, z_{1}\right)$ and at the bottom of the stratum $\left(\rho_{2}, z_{2}\right)$ are known, the values 


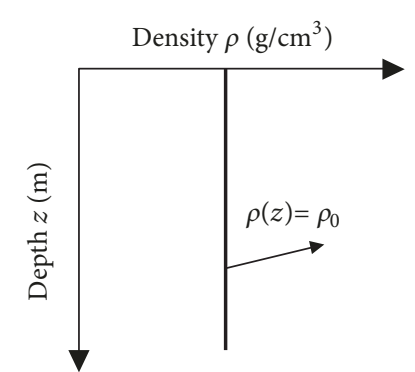

(a)

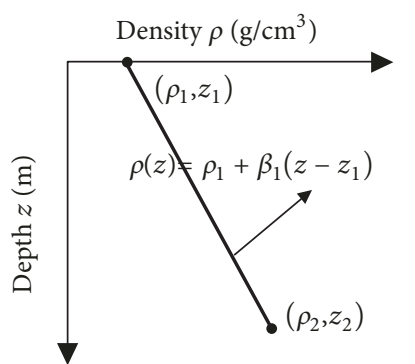

(b)

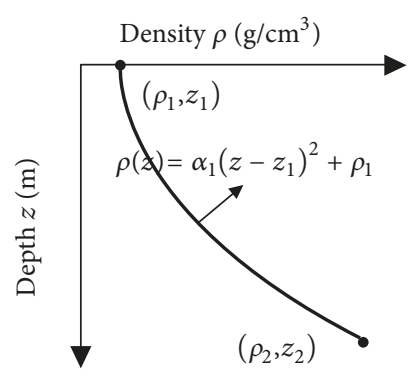

(c)

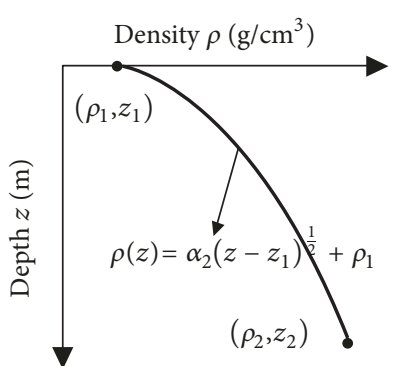

(d)

FIGURE 5: Assumed density profiles in the ground: (a) constant; (b) linear; (c) parabolic; (d) square root.

of $\beta_{1}, \alpha_{1}$, and $\alpha_{2}$ in (11), (12), and (13) can be determined as follows:

$$
\begin{aligned}
& \beta_{1}=\frac{\left(\rho_{2}-\rho_{1}\right)}{\left(z_{2}-z_{1}\right)} \\
& \alpha_{1}=\frac{\left(\rho_{2}-\rho_{1}\right)}{\left(z_{2}-z_{1}\right)^{2}} \\
& \alpha_{2}=\frac{\left(\rho_{2}-\rho_{1}\right)}{\left(z_{2}-z_{1}\right)^{0.5}}
\end{aligned}
$$

Eqs. (17), (18), (19), and (20) can be used to calculate $R_{\rho}$ when the density functions are constant, linear, parabolic, and square root, respectively.

$$
R_{\rho}=\frac{3\left(A \rho_{0}^{2}-B \rho_{0}+C\right)}{4 b^{3}} \int\left[b^{2}-\left(z-z_{i}\right)^{2}\right] \delta z
$$

$$
\begin{aligned}
R_{\rho} & =\frac{3}{4 b^{3}} \int\left\{A\left[\rho_{1}+\beta\left(z-z_{1}\right)\right]^{2}\right. \\
& \left.-B\left[\rho_{1}+\beta\left(z-z_{1}\right)\right]+C\right\}\left[b^{2}-\left(z-z_{i}\right)^{2}\right] \delta z \\
R_{\rho} & =\frac{3}{4 b^{3}} \int\left\{A\left[\alpha_{1}\left(z-z_{1}\right)^{2}+\rho_{1}\right]^{2}\right. \\
& \left.-B\left[\alpha_{1}\left(z-z_{1}\right)^{2}+\rho_{1}\right]+C\right\}\left[b^{2}-\left(z-z_{i}\right)^{2}\right] \delta z \\
R_{\rho} & =\frac{3}{4 b^{3}} \int\left\{A\left[\alpha_{2}\left(z-z_{1}\right)^{1 / 2}+\rho_{1}\right]^{2}\right. \\
& \left.-B\left[\alpha_{2}\left(z-z_{1}\right)^{1 / 2}+\rho_{1}\right]+C\right\}\left[b^{2}-\left(z-z_{i}\right)^{2}\right] \delta z
\end{aligned}
$$

The solutions for (17) to (20) are derived in (21) to (24), respectively.

$$
\left.\begin{array}{c}
R_{\rho}=\left.\frac{3}{4 b^{3}}\left(A \rho_{0}^{2}-B \rho_{0}+C\right)\left\{-\frac{z^{3}}{3}+z_{i} z^{2}+\left(b^{2}-z_{i}^{2}\right) z\right\}\right|_{z_{1}} ^{z_{2}} \\
R_{\rho}=\frac{3}{4 b^{3}}\left\{\begin{array}{c}
\frac{-A \beta^{2} z^{5}}{5}+\frac{\left(-2 A \rho_{1} \beta+2 A z_{i} \beta^{2}+2 A \beta^{2} z_{1}+B \beta\right) z^{4}}{4} \\
+\frac{\left(\begin{array}{c}
b^{2} A \beta^{2}-A \rho_{1}^{2}+4 A z_{i} \rho_{1} \beta-A z_{i}^{2} \beta^{2}+2 A \rho_{1} \beta z_{1}-A \beta^{2} z_{1}^{2}-4 A z_{i} \beta^{2} z_{1} \\
+B \rho_{1}-B \beta z_{1}-2 B z_{i} \beta-C
\end{array}\right) z^{3}}{3} \\
+\left(\begin{array}{c}
\frac{2 b^{2} A \beta \rho_{1}-2 b^{2} A \beta^{2} z_{1}-2 A \rho_{1} \beta z_{i}^{2}+2 A z_{i} \rho_{1}^{2}-4 A z_{i} \rho_{1} \beta z_{1}+2 A z_{i} \beta^{2} z_{1}^{2}}{+2 A z_{i}^{2} \beta^{2} z_{1}-b^{2} B \beta+B z_{i}^{2} \beta-2 B z_{i} \rho_{1}+2 B z_{i} \beta z_{1}+2 C z_{i}} \\
2
\end{array}\right) \\
+\left(\begin{array}{c}
b^{2} A \rho_{1}^{2}- \\
2 b^{2} A \rho_{1} \beta z_{1}+b^{2} A \beta^{2} z_{1}^{2}-A z_{i}^{2} \rho_{1}^{2}+2 A \rho_{1} \beta z_{1} z_{i}^{2}-A z_{i}^{2} \beta^{2} z_{1}^{2} \\
-b^{2} B \rho_{1}+b^{2} B \beta z_{1}+B z_{i}^{2} \rho_{1}-B z_{i}^{2} \beta z_{1}+C b^{2}-C z_{i}^{2}
\end{array}\right.
\end{array}\right\} z z^{2}
\end{array}\right\}\left.\right|_{z_{1}} ^{z_{2}}
$$




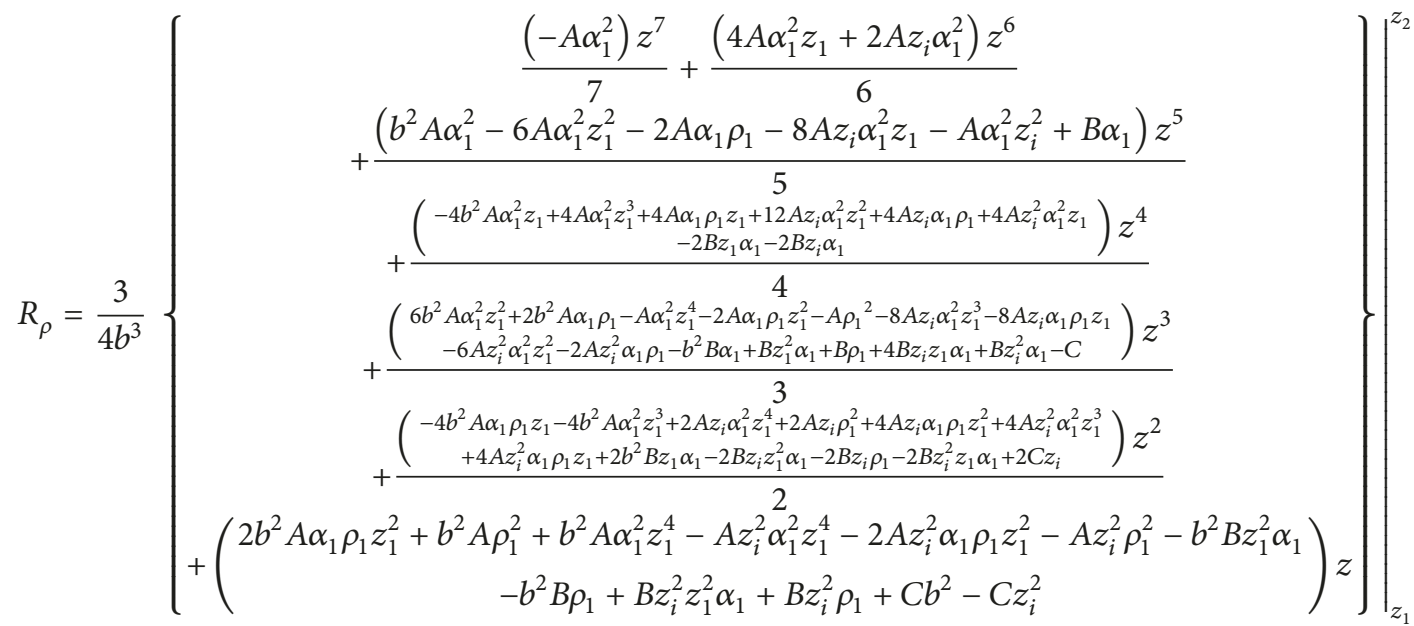

$$
\begin{aligned}
& R_{\rho}=\left.\frac{3}{4 b^{3}}\left\{\begin{array}{c}
-\frac{1}{4} A \alpha_{2}^{2}\left(z-z_{1}\right)^{4}+\frac{2}{7}\left(B \alpha_{2}-2 A \rho_{1} \alpha_{2}\right)\left(z-z_{1}\right)^{7 / 2} \\
+\frac{1}{3}\left[-2 A \alpha_{2}^{2}\left(z_{1}-z_{i}\right)\right]\left(z-z_{1}\right)^{3}-\frac{1}{3}\left(A \rho_{1}^{2}-B \rho_{1}\right)\left(z-z_{i}\right)^{3}-\frac{1}{3} C z^{3} \\
+\frac{2}{5}\left[2 B \alpha_{2}\left(z_{1}-z_{i}\right)-4 A \rho_{1} \alpha_{2}\left(z_{1}-z_{i}\right)\right]\left(z-z_{1}\right)^{5 / 2} \\
+\frac{1}{2}\left[b^{2} A \alpha_{2}^{2}-A \alpha_{2}^{2}\left(z_{1}-z_{i}\right)^{2}\right]\left(z-z_{1}\right)^{2}+C z_{i} z^{2} \\
+\frac{2}{3}\left[2 b^{2} A \rho_{1} \alpha_{2}-2 A \rho_{1} \alpha_{2}\left(z_{1}-z_{i}\right)^{2}-b^{2} B \alpha_{2}+B \alpha_{2}\left(z_{1}-z_{i}\right)^{2}\right]\left(z-z_{1}\right)^{3 / 2} \\
+\left[b^{2} A \rho_{1}^{2}-b^{2} B \rho_{1}+C\left(b^{2}-z_{i}^{2}\right)\right] z
\end{array}\right\}\right|_{z} ^{z_{2}}
\end{aligned}
$$

If the gamma ray source and detector are in strata that can be described by one function of density, $R_{\rho}$ is calculated as an integral of that function. If the gamma ray source and detector are in strata described by two or more functions, $R_{\rho}$ is calculated as a piecewise integration of those functions. Then, the composite density $\left(\rho_{\mathrm{c}}\right)$, which is referenced to the midpoint of the spheroid, can be calculated by (25). Finally, the $\rho_{c}$ profile can be obtained as the midpoint of the spheroid moves with depth along with the penetration of the ND-CP.

$$
\rho_{c}=\frac{4.0954-\sqrt{2.5056 R_{\rho}-0.3715}}{1.2528}
$$

3.3. Verification of the Theoretical Model. The ND-CP was used to measure the density profiles of two kinds of layered soils by Karthikeyan and Tan in the laboratory [29]. The experimental setup consists of a loading frame and a cubic steel tank with dimensions of $1.5 \mathrm{~m} \times 1.5 \mathrm{~m} \times 1.5 \mathrm{~m}$. The loading frame is placed on top of the steel tank to push the ND-CP using a hydraulic piston. The ND-CP measurement and the depth meter readings were recorded through a data logger. Figure 6 shows schematic configurations for layered soils in the steel tank. One configuration is with a top layer of water and bottom layer of soil (Figure 6(a)), and the other configuration is with a top layer of kaolin clay slurry and bottom layer of soil (Figure 6(b)). The measured $\rho_{\mathrm{c}}$ profiles do not show a sharp transition at the interface of the water and soil layers (Figure 6(a)) or at the interface of the slurry and soil layers (Figure 6(b)). The change in density from the water/slurry to the soil is extended over a distance equal to the source-detector separation $(2 b)$. This transition occurs as the gamma ray source enters the soil layer and continues until the gamma ray detector enters the soil layer. The actual $\rho$ profile shows a step change in density, while the measured $\rho_{\text {c }}$ profile shows a continuous change in density. The change pattern in the measured $\rho_{\mathrm{c}}$ profile indicates that the ND-CP is penetrating the interface of two layers with different density values. The $\rho_{\mathrm{c}}$ profiles predicted by the theoretical model are also shown in the figure, and these predicted results show a close agreement with the measured $\rho_{\mathrm{c}}$ profiles, suggesting that the theoretical model is able to describe the signature of the $\rho_{\mathrm{c}}$ profiles measured by the ND-CP. The theoretical model will be used to investigate the differences between the actual $\rho$ profiles in the ground and the measured $\rho_{\mathrm{c}}$ profiles by the ND-CP.

\section{Method for Deducing Actual $\rho$ Profile}

4.1. Comparison of $\rho$ and $\rho_{c}$ Profiles. The density profiles of complex strata, which are common in engineering practice, are assumed in Figure 7. Figure 7(a) shows the density profiles of layered strata. The density of the first layer is $1.6 \mathrm{~g} / \mathrm{cm}^{3}$, and the densities of the second layer are $1.5 \mathrm{~g} / \mathrm{cm}^{3}, 1.7 \mathrm{~g} / \mathrm{cm}^{3}$, or $1.8 \mathrm{~g} / \mathrm{cm}^{3}$. Figure 7 (b) shows the density profiles of strata with soft interlayers. The density of the strata is $1.7 \mathrm{~g} / \mathrm{cm}^{3}$. Three different soft interlayers are assumed. For the first case, the soft interlayer has a density of $1.3 \mathrm{~g} / \mathrm{cm}^{3}$ and a thickness of $0.15 \mathrm{~m}$; for the second case, the density is $1.5 \mathrm{~g} / \mathrm{cm}^{3}$ and 


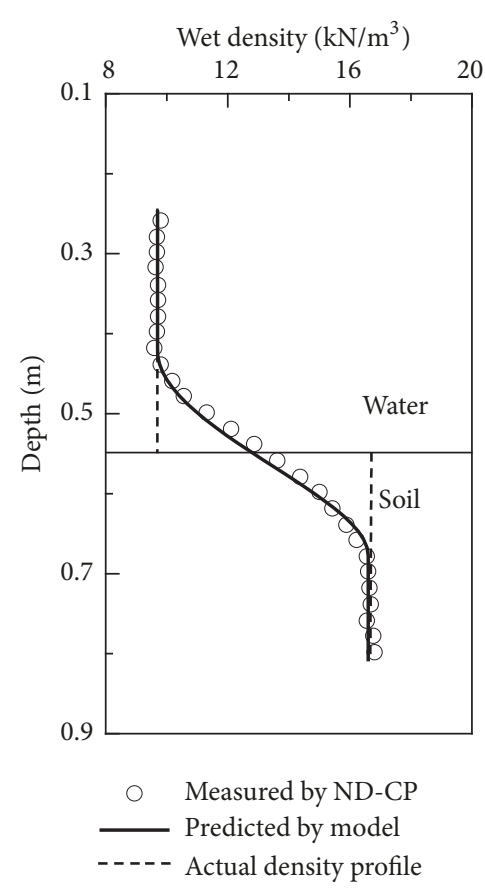

(a)

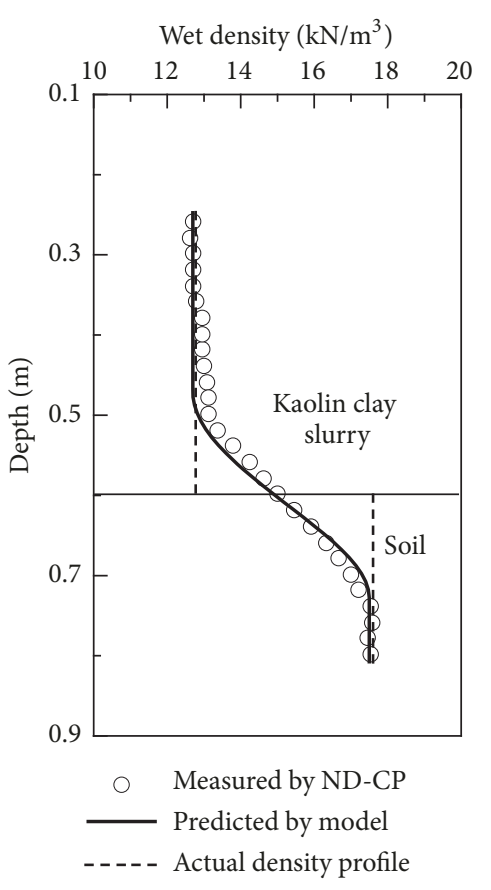

(b)

FIGURE 6: Verification of the theoretical model with laboratory test results.

the thickness is $0.15 \mathrm{~m}$; for the third case, the density is $1.3 \mathrm{~g} / \mathrm{cm}^{3}$ and the thickness is $0.3 \mathrm{~m}$. Figure $7(\mathrm{c})$ shows the density profiles of bed sediments. The density of the water is $1.0 \mathrm{~g} / \mathrm{cm}^{3}$, and the density of the bed mud increases with depth in a linear fashion. Three different layers of fluid mud are assumed. For the first case, the fluid mud has a maximum density of $1.2 \mathrm{~g} / \mathrm{cm}^{3}$ and thickness of $0.2 \mathrm{~m}$; for the second case, the maximum density is $1.3 \mathrm{~g} / \mathrm{cm}^{3}$ and the thickness is $0.2 \mathrm{~m}$; for the third case, the maximum density is $1.15 \mathrm{~g} / \mathrm{cm}^{3}$ and the thickness is $0.25 \mathrm{~m}$.

The $\rho_{\mathrm{c}}$ profiles are calculated by the theoretical model when the ND-CP penetrates into the assumed complex strata (Figure 7 ), and the results are compared with the $\rho$ profiles. The $\rho_{\mathrm{c}}$ profiles are clearly different from the $\rho$ profiles near the interface of the layered strata, near the soft interlayer, and near the upper and lower boundaries of the fluid mud. The differences between $\rho_{\mathrm{c}}$ and $\rho$ near the interface of the layered strata in Figure 7(a) are explained as follows. (1) When both the gamma ray source and detector are in the top layer, $\rho_{\mathrm{c}}$ is equal to the density of the top layer. (2) Once the gamma ray source enters the bottom layer, $\rho_{c}$ will be the weighted average density of the two layers, while $\rho$ is still the density of the first layer. (3) When the midpoint of the gamma ray source and detector enters the bottom layer, $\rho$ will change to the density of the bottom layer, while $\rho_{\mathrm{c}}$ is still the weighted average density of the two layers because the gamma ray detector is still in the first layer. (4) When the gamma ray detector also enters the bottom layer, $\rho_{c}$ is equal to $\rho$ of the bottom layer. Similar to the profiles for the case in which the ND-CP passes through the interface of layered strata, the $\rho_{\mathrm{c}}$ profiles are different from the $\rho$ profiles when the ND-CP passes through a soft interlayer and the fluid mud. Therefore, the $\rho_{\mathrm{c}}$ profile measured by the ND-CP must be interpreted, and the actual $\rho$ profile must be deduced from the measured $\rho_{\mathrm{c}}$ profile.

4.2. Method for Deducing Actual $\rho$ Profile from Measured $\rho_{c}$ Profile. A method for deducing the actual $\rho$ profile from the measured $\rho_{\mathrm{c}}$ profile is proposed as follows:

(1) Determining the depths of and densities at the boundaries of each stratum based on the features of the measured $\rho_{c}$ profile. (2) Assuming the probable density function of each stratum and the probable $\rho$ profile of the strata. (3) Back-calculating the $\rho_{\mathrm{c}}$ profile by using the theoretical model for the case of the ND-CP passing through layered strata. (4) Comparing the calculated $\rho_{\mathrm{c}}$ profile with the measured $\rho_{c}$ profile and adjusting the assumed $\rho$ profile until the calculated $\rho_{\mathrm{c}}$ profile matches the measured $\rho_{\mathrm{c}}$ profile well. (5) Determining the actual $\rho$ profile to be the assumed $\rho$ profile when the calculated $\rho_{\mathrm{c}}$ profile fits the measured $\rho_{\mathrm{c}}$ profile.

For the layered strata, as shown in Figure $7(\mathrm{a})$, the measured $\rho_{\mathrm{c}}$ profile can be divided into three sections, namely, the first constant section, the second curved section, and the third constant section. The measured $\rho_{\mathrm{c}}$ values are different from the actual $\rho$ values in the range of $2 b$, which is the distance between the gamma ray source and detector. From the $\rho_{\mathrm{c}}$ profile, the boundary between layered strata can be determined, i.e., the midpoint of the second curved section of the $\rho_{\mathrm{c}}$ profile. The actual $\rho$ value of the top layer is assumed to be the measured $\rho_{\mathrm{c}}$ value of the first constant section, and the actual $\rho$ value of the bottom layer is assumed to be the measured $\rho_{\mathrm{c}}$ value of the third constant section. The $\rho_{\mathrm{c}}$ profile 


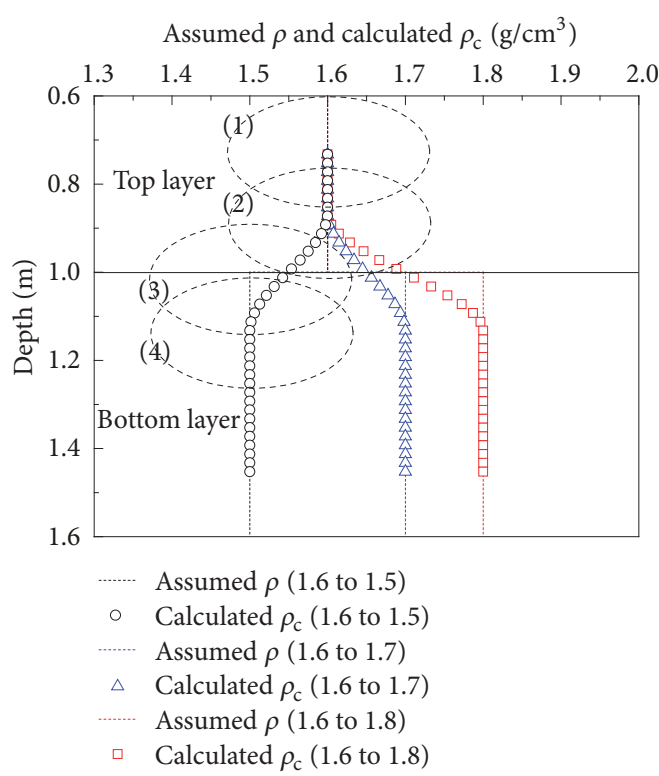

(a) Layered strata

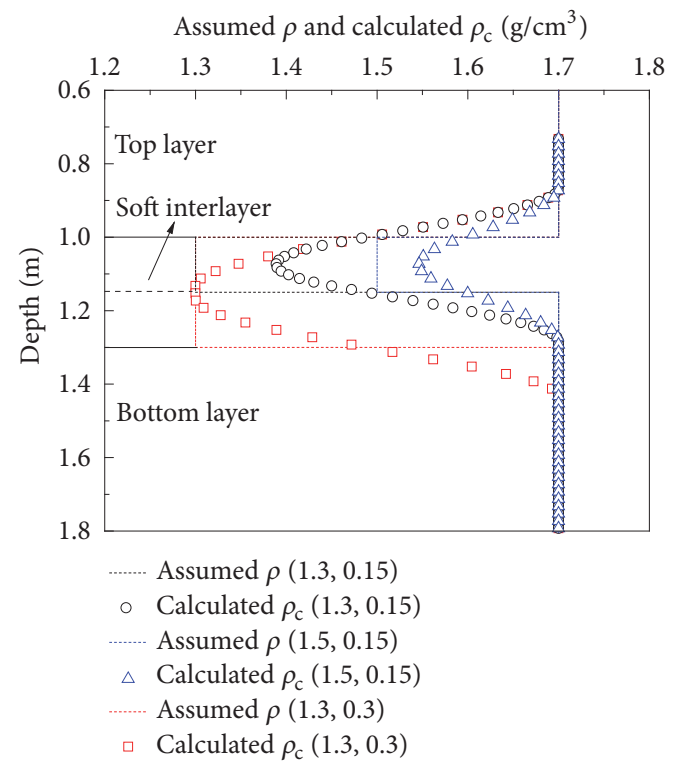

(b) Strata with soft interlayers

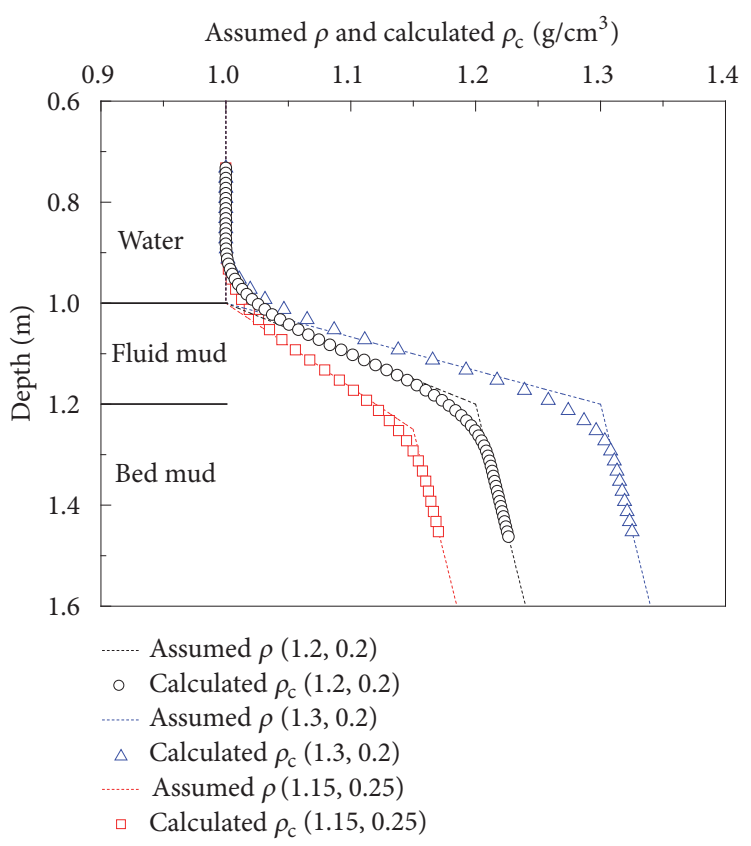

(c) Bed sediments

FIGURE 7: Comparison of assumed $\rho$ and calculated $\rho_{\mathrm{c}}$ profiles.

is back-calculated assuming that the ND-CP passes through the layered strata. If the calculated $\rho_{\mathrm{c}}$ profile matches well with the measured $\rho_{\mathrm{c}}$ profile, the actual $\rho$ profile of the layered strata was determined.

For the strata with a soft interlayer, as shown in Figure $7(\mathrm{~b})$, the measured $\rho_{\mathrm{c}}$ profile can be divided into three sections, namely, the first constant section, the second curved section, and the third constant section. The measured $\rho_{\mathrm{c}}$ values are different from the actual $\rho$ values near the soft interlayer ( $2 b$ plus the thickness of the soft interlayer). From the $\rho_{\mathrm{c}}$ profile, the upper and lower boundaries of the soft interlayer can be determined, that is, the depth of the start point of the second curved section plus $b$ and the depth of the end point of the second curved section minus $b$. The actual $\rho$ of the top layer is assumed to be the measured $\rho_{\mathrm{c}}$ of the first constant section, and the actual $\rho$ of the bottom layer is assumed to be the measured $\rho_{\mathrm{c}}$ of the third constant section. The actual $\rho$ of the soft interlayer can be assumed based on the minimum $\rho_{\mathrm{c}}$ in the second curved section. The $\rho_{\mathrm{c}}$ profile is back-calculated assuming that the ND-CP passes through 


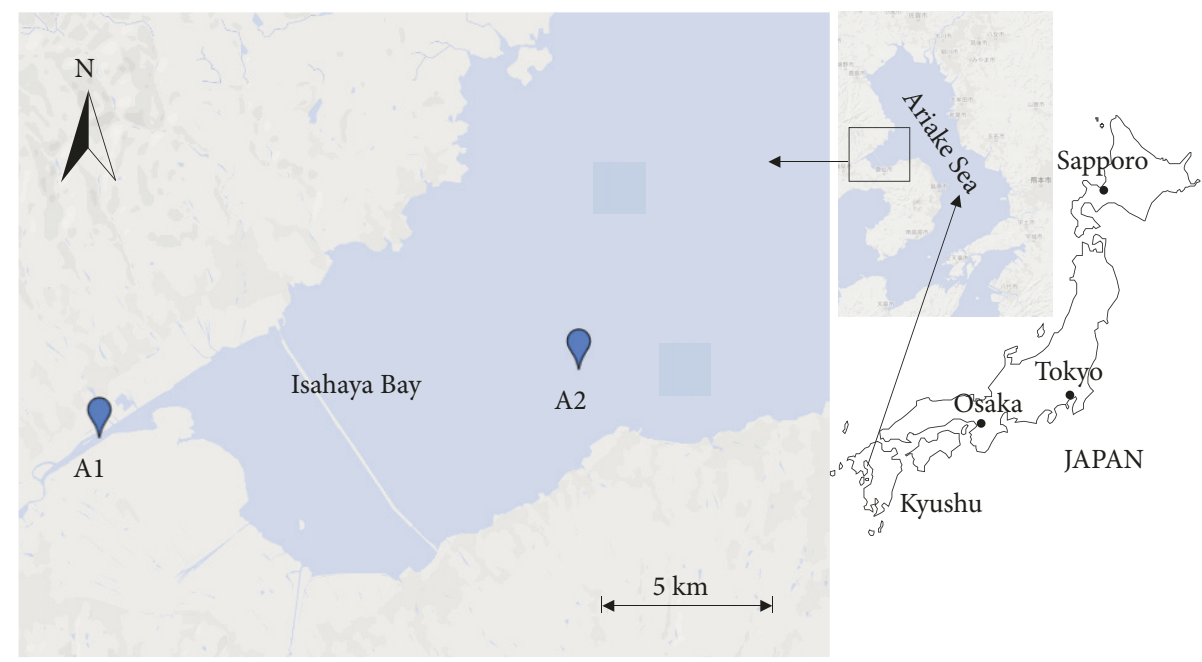

FIGURE 8: Investigation locations.

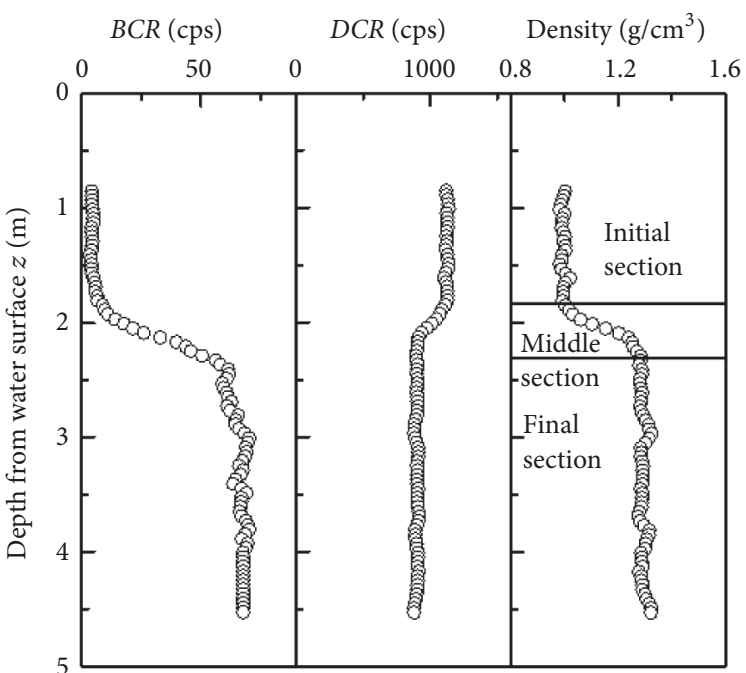

(a)

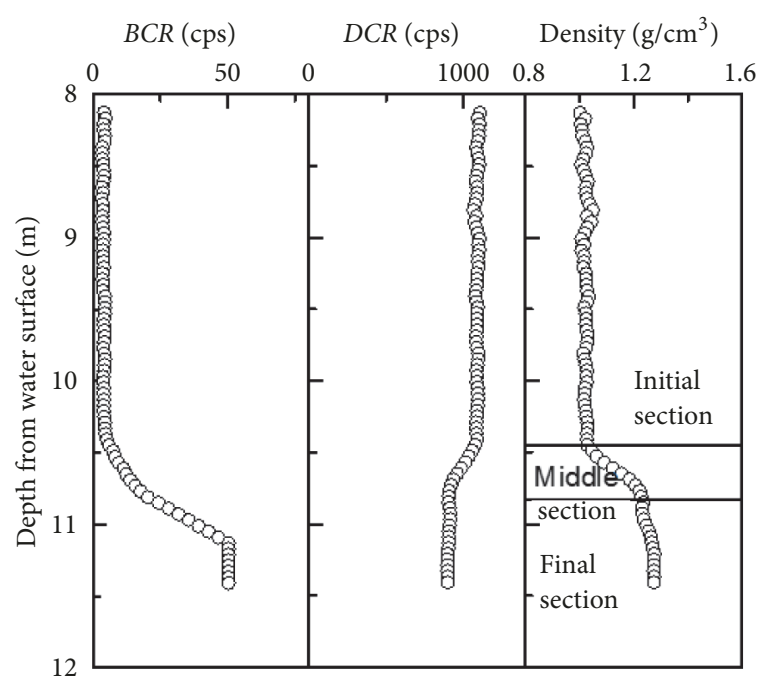

(b)

FIGURE 9: Investigation results: (a) at location A1; (b) at location A2.

the strata with a soft interlayer. The actual $\rho$ profile of strata with a soft interlayer is determined when the calculated $\rho_{\mathrm{c}}$ profile matches the measured $\rho_{\mathrm{c}}$ profile well.

For bed sediments, as shown in Figure 7(c), the measured $\rho_{\mathrm{c}}$ profile can be divided into three sections, namely, the first constant section, the second curved section, and the third linear section. The measured $\rho_{\mathrm{c}}$ values are different from the actual $\rho$ values near the upper and lower boundaries of the fluid mud. From the $\rho_{\mathrm{c}}$ profile, the upper and lower boundaries of the fluid mud can be determined; that is, the depth of the point from which density increases plus $b$ and the depth of the point from which density slightly increases linearly minus $b$. The actual $\rho$ of the water layer is assumed to be the measured $\rho_{\mathrm{c}}$ of the first constant section. The slope of the actual $\rho$ profile of the bed mud layer is assumed to be the slope of the measured $\rho_{\mathrm{c}}$ profile of the third linear section. The actual $\rho$ distribution of the fluid mud layer can be assumed to be a parabolic, linear, or square root function. The $\rho_{\mathrm{c}}$ profile is back-calculated assuming that the ND-CP passes through bed sediments. The actual $\rho$ profile of bed sediments can be determined when the calculated $\rho_{\mathrm{c}}$ profile fits the measured $\rho_{\mathrm{c}}$ profile.

\section{An Example of Practical Application}

5.1. ND-CP Measured $\rho_{c}$ Profile. The density profiles of bed sediments at Isahaya Bay, Japan, were investigated using the ND-CP. The details on the equipment and methods used in this investigation can be found in a prior publication [23]. The ND-CP measured density profiles at two locations (Figure 8) are used to illustrate how to use the proposed method to determine the actual $\rho$ profile from the measured $\rho_{\mathrm{c}}$ profile.

The measured $B C R$ and $D C R$ and the calculated $\rho_{\mathrm{c}}$ values at locations $A 1$ and $A 2$ are shown in Figure 9. BCR 


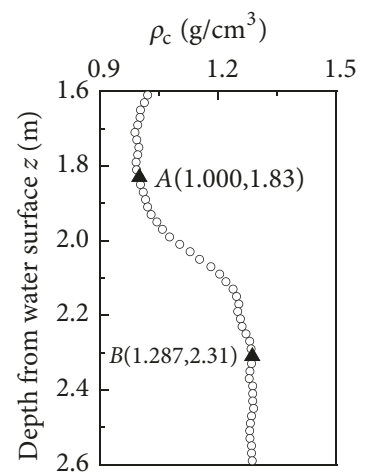

(a)

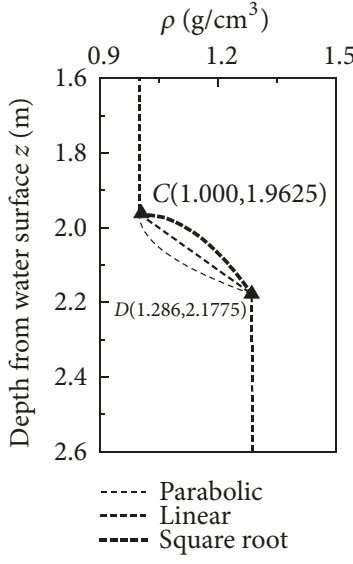

(b)

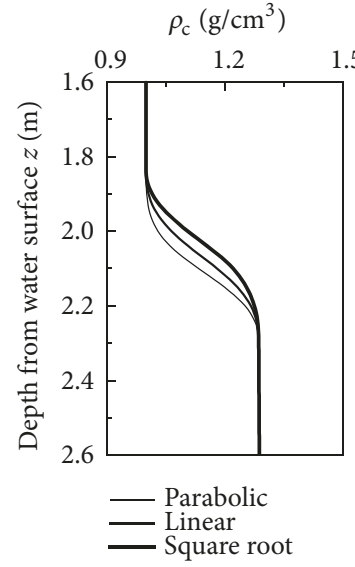

(c)

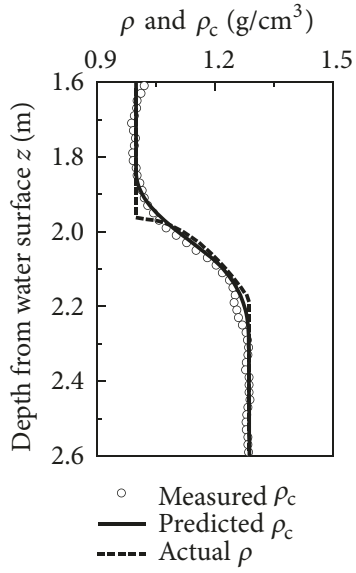

(d)

FIGURE 10: Determination of the actual density profile of bed sediments at location A1: (a) measured $\rho_{\mathrm{c}}$ profile; (b) assumed $\rho$ profile; (c) calculated $\rho_{\mathrm{c}}$ profile; (d) deduced $\rho$ profile.

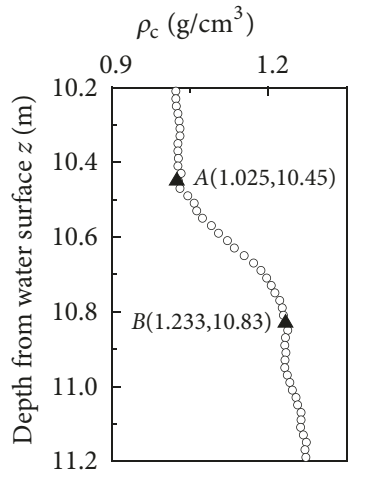

(a)

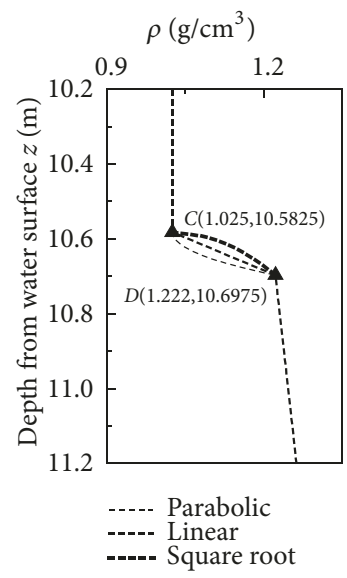

(b)

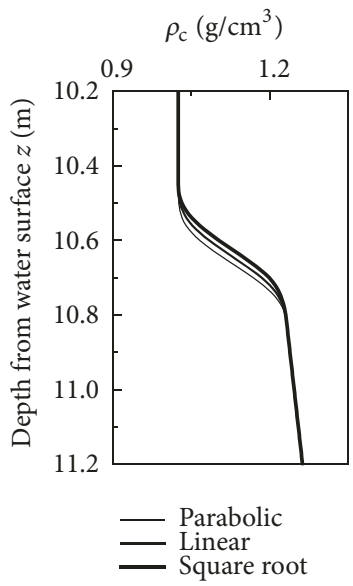

(c)

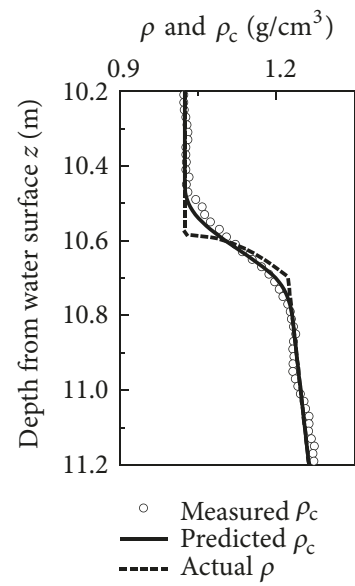

(d)

FIGURE 11: Determination of the actual density profile of bed sediments at location A2: (a) measured $\rho_{\mathrm{c}}$ profile; (b) assumed $\rho$ profile; (c) calculated $\rho_{\mathrm{c}}$ profile; (d) deduced $\rho$ profile.

is approximately zero in water and approximately $50 \mathrm{cps}$ in bed mud. DCR in bed sediments is lower than that in water and decreases with increasing density. $R_{\rho}$ can be calculated according to the measured $B C R$ and $D C R$ by using (1), and then the density can be calculated by using (25). The density profiles show similar transitions and vary significantly around the water/mud interface. The measured $\rho_{\text {c }}$ profiles can be divided into three sections, namely, an initial constant section, a middle curved section, and a final linear section.

5.2. Determination of the Actual $\rho$ Profile. There is an obvious difference between the actual $\rho$ and the measured $\rho_{\mathrm{c}}$ profiles near the upper and lower boundaries of the fluid mud layer, as shown in Figure 7 (c). The theoretical model proposed in Section 3 and the method proposed in Section 4 were used to deduce the actual $\rho$ profiles from the measured $\rho_{\mathrm{c}}$ profiles as follows.
(1) For the measured $\rho_{\mathrm{c}}$ profile at location A1 (Figure $10(\mathrm{a}))$, the $\rho_{\mathrm{c}}$ at and depth of the intersection of the initial constant and middle curved sections (point $A$ ) are $1.000 \mathrm{~g} / \mathrm{cm}^{3}$ and $1.83 \mathrm{~m}$, respectively. The $\rho_{\mathrm{c}}$ at and depth of the intersection of the middle curved and final linear sections (point $B$ ) are $1.287 \mathrm{~g} / \mathrm{cm}^{3}$ and $2.31 \mathrm{~m}$, respectively. For the measured $\rho_{\mathrm{c}}$ profile at location A2 (Figure 11(a)), $\rho_{\mathrm{c}}$ and depth at point $A$ are $1.025 \mathrm{~g} / \mathrm{cm}^{3}$ and $10.45 \mathrm{~m}$, respectively. $\rho_{\mathrm{c}}$ and depth at point $B$ are $1.233 \mathrm{~g} / \mathrm{cm}^{3}$ and $10.83 \mathrm{~m}$, respectively.

(2) For the assumed $\rho$ profile at location A1 (Figure 10(b)), the depth of the upper boundary of the fluid mud at point $C$ is $1.9625 \mathrm{~m}$, which is equal to the depth at point $A(1.83 \mathrm{~m})$ plus $b$ $(0.1325 \mathrm{~m})$. The depth of the lower boundary of the fluid mud at point $D$ is $2.1775 \mathrm{~m}$, which is equal to the depth at point $B$ $(2.31 \mathrm{~m})$ minus $b(0.1325 \mathrm{~m}) . \rho$ at point $C$ is $1.000 \mathrm{~g} / \mathrm{cm}^{3}$, which is equal to $\rho_{\mathrm{c}}$ at point $A . \rho$ at point $D$ is $1.286 \mathrm{~g} / \mathrm{cm}^{3}$, which is equal to $\rho_{\mathrm{c}}$ at point $B$ minus $k b$ (where $k$ is the slope of the final linear section). For the assumed $\rho$ profile at location A2 
(Figure 11(b)), the depth at point $C$ is $10.5825 \mathrm{~m}$, and the depth at point $D$ is $10.6975 \mathrm{~m} . \rho$ at point $C$ is $1.025 \mathrm{~g} / \mathrm{cm}^{3}$, and $\rho$ at point $D$ is $1.222 \mathrm{~g} / \mathrm{cm}^{3}$.

(3) The $\rho$ function of the first section (water layer) is assumed to be constant, the $\rho$ function of the second section (fluid mud) is assumed to be parabolic, linear, or square root, and the $\rho$ function of the third section (bed mud) is assumed to be linear. The $\rho$ of the first section is assumed to be 1.000 $\mathrm{g} / \mathrm{cm}^{3}$ (freshwater) and $1.025 \mathrm{~g} / \mathrm{cm}^{3}$ (seawater) for $\rho$ profiles at locations A1 and A2, respectively. The parabolic, linear, and square root density functions for the second section can be determined according to the density and depth values at point $C$ and point $D$ by (11) to (16). The slope of the third section is assumed to be 0.005 and 0.08 , respectively, for the $\rho$ profiles at locations $\mathrm{A} 1$ and $\mathrm{A} 2$ based on the measured $\rho_{\mathrm{c}}$ values at the final linear sections.

(4) The $\rho_{\mathrm{c}}$ profiles are calculated by (21) to (25), assuming the ND-CP passes through the strata with the assumed $\rho$ profiles in Figures 10(b) and 11(b). The calculated $\rho_{\mathrm{c}}$ profiles at locations A1 and A2 are shown in Figures 10(c) and 11(c), respectively. The calculated $\rho_{\mathrm{c}}$ profiles clearly vary when the assumed $\rho$ function of the second section varies (among parabolic, linear, or square root functions). Therefore, the calculated $\rho_{\mathrm{c}}$ profile can be changed by adjusting the assumed $\rho$ profile.

(5) The calculated $\rho_{\mathrm{c}}$ profiles for the different assumed $\rho$ profiles are compared with the measured $\rho_{c}$ profiles. The calculated $\rho_{\mathrm{c}}$ profiles match the measured $\rho_{\mathrm{c}}$ profiles well if the density function of the second section is assumed to be a square root function [24]. The deduced $\rho$ profiles are also shown in Figures $10(\mathrm{~d})$ and $11(\mathrm{~d})$. The $\rho$ values are clearly different from the $\rho_{\mathrm{c}}$ values around the fluid mud layer. Therefore, it is suggested that the deduced $\rho$ profile from the measured $\rho_{\text {c }}$ profile should be adopted to predict sediment transport, determine nautical depth, and plan dredging projects.

\section{Summary and Conclusions}

The nuclear density cone penetrometer (ND-CP) can be used for in situ investigation on the soil density, while it measures the composite density $\left(\rho_{\mathrm{c}}\right)$ of the soil within a spheroid centered at the midpoint between the gamma ray source and the detector. A theoretical model for calculating $\rho_{\mathrm{c}}$ and a method for deducing the $\rho$ profile from the measured $\rho_{\mathrm{c}}$ profile were proposed in this study. The following conclusions can be drawn from this study.

(1) A theoretical model for predicting the count rate ratio $\left(R_{\rho}\right)$ of the ND-CP is proposed, and equations for calculating $R_{\rho}$ are derived for the case of the ND-CP penetrating into strata with different density distribution functions. The calculated $\rho_{\mathrm{c}}$ profiles exhibit a good fit to the laboratorymeasured $\rho_{\mathrm{c}}$ profiles by the ND-CP, indicating that the proposed theoretical model can be used to calculate the $\rho_{\mathrm{c}}$ within the spheroid. A comparison of the $\rho$ and $\rho_{\mathrm{c}}$ values shows that the $\rho_{\mathrm{c}}$ profiles are considerably different from the actual $\rho$ profiles at the boundaries of each stratum where the density changes suddenly.
(2) A method for deducing the actual $\rho$ profile from the measured $\rho_{\mathrm{c}}$ profile is proposed according to the differences between the $\rho$ and $\rho_{\mathrm{c}}$ profiles. The depth of and density at the boundaries of each stratum are first determined based on the features of the measured $\rho_{\mathrm{c}}$ profile. Then, the $\rho$ profile of the strata is assumed, and the $\rho_{\mathrm{c}}$ profile is back-calculated. Finally, the calculated $\rho_{\mathrm{c}}$ profile is compared with the measured $\rho_{\mathrm{c}}$ profile, and the actual $\rho$ profile is identified when the calculated $\rho_{\mathrm{c}}$ profile fits the measured $\rho_{\mathrm{c}}$ profile.

(3) The proposed model and method are used to determine the actual $\rho$ profiles from measured $\rho_{c}$ profiles of bed sediments in the field. The actual $\rho$ values are clearly different from the $\rho_{\mathrm{c}}$ values around the fluid mud layer. It is suggested that the deduced $\rho$ profile should be adopted to predict sediment transport, determine nautical depth, and plan dredging projects. The findings in this study would be beneficial for the in situ determination of density profiles using the ND-CP. Further research should be conducted to verify the proposed model and method in determining the actual $\rho$ profiles from the measured $\rho_{\mathrm{c}}$ profiles by the ND-CP in the field of geotechnical and pavement engineering.

\section{Acronyms}

a: $\quad$ Equatorial radius of the spheroid

2b: Gamma ray source-detector separation distance

A: $\quad$ Constant 0.6264

B: $\quad$ Constant 4.0954

C: $\quad$ Constant 6.8422

BCR: Background count rate

DCR: Density count rate

SCR: $\quad$ Standard count rate

$k$ : $\quad$ Slope of the final linear region

ND-CP: Nuclear density cone penetrometer

$R_{\rho}: \quad$ Count rate ratio

$V: \quad$ Volume of spheroid

$z: \quad$ Depth

$z_{\mathrm{i}}$ : Depth corresponding to the midpoint of the ellipse

$z_{1}$ : $\quad$ Depth at the top of stratum

$z_{2}$ : Depth at the bottom of stratum

$\alpha_{1}$ : $\quad$ Fitting parameter for parabolic density distribution

$\alpha_{2}$ : $\quad$ Fitting parameter for square root density

distribution

$\beta_{1}$ : $\quad$ Fitting parameter for linear density

distribution

$\rho: \quad$ Density

$\rho_{\mathrm{c}}: \quad$ Composite density

$\rho_{0}$ : Density value of constant density

distribution

$\rho_{1}$ : $\quad$ Density value at the top of stratum

$\rho_{2}$ : $\quad$ Density value at the bottom of stratum

$\Delta R_{\rho}$ : Count rate ratio from a small volume

$\delta z: \quad$ Differential of $z$

$\partial V: \quad$ Differential of $V$

$\partial R_{\rho}: \quad$ Differential of count rate ratio. 


\section{Data Availability}

The data used to support the findings of this article are available from the authors upon request.

\section{Conflicts of Interest}

The authors declare that they have no competing interests.

\section{Acknowledgments}

The authors would like to acknowledge the financial support of the National Key Research and Development Program of China (Grant No. 2017YFC0805402), the Major Program of National Natural Science Foundation of China (Grant No. 51890911), and the National Natural Science Foundation of China (Grant No. 51509181).

\section{References}

[1] K. Terzaghi, R. B. Peck, and G. Mesri, Soil Mechanics in Engineering Practice, Wiley, New York, NY, USA, 3rd edition, 1996.

[2] Y. Wu, H. Lyu, J. Han, and S. Shen, "Dewatering-induced building settlement around a deep excavation in soft deposit in Tianjin, China," Journal of Geotechnical and Geoenvironmental Engineering, vol. 145, no. 5, Article ID 05019003, pp. 1-14, 2019.

[3] D. Sarkar and A. Haldar, Physical and Chemical Methods in Soil Analysis- Fundamental Concepts of Analytical Chemistry and Instrumental Techniques, New Age International Ltd., New Delhi, India, 2005.

[4] S.-J. Zheng, "The new method for engineering geologic investigation-Nuclear density cone penetrometer," Underground Engineering and Tunnels, vol. 4, no. 2, pp. 16-21, 1995 (Chinese).

[5] S. Horpibulsuk, W. Katkan, and A. Apichatvullop, "An approach for assessment of compaction curves of fine grained soils at various energies using a one point test," Soils and Foundations, vol. 48 , no. 1 , pp. $115-125,2008$.

[6] W. H. McAnally, C. Friedrichs, D. Hamilton et al., "Management of fluid mud in estuaries, bays, and lakes. I: Present state of understanding on character and behavior," Journal of Hydraulic Engineering, vol. 133, no. 1, pp. 9-22, 2007.

[7] D. Campbell and J. Kenneth Henshall, "Bulk density," in Soil and Environmental Analysis-Physical Methods, K. A. Smith and C. E. Mullins, Eds., pp. 315-348, Marcel Dekker, Inc, New York, NY, USA, 2nd edition, 2000.

[8] National Energy Administration of China, DL 5270-2012: Test Code for Density and Moisture by Nuclear Methods, China Electric Power Press, Beijing, China, 2012.

[9] B. Pontecorvo, "Neutron well logging," Oil Gas Journal, vol. 40, pp. 32-33, 1941.

[10] J. Homilius and S. Lorch, "On the theory of gamma ray scattering in boreholes," Geophysical Prospecting, vol. 6, no. 4, pp. 342-364, 1958.

[11] A. C. Meigh and B. O. Skipp, "Gamma-ray and neutron methods of measuring soil density and moisture," Géotechnique, vol. 10, no. 3, pp. 110-126, 1960.
[12] P. A. Ruygrok, "Evaluation of the gamma and neutron radiation scattering and transmission methods for soil density and moisture determination," Geotechnical Testing Journal, vol. 11, no. 1, pp. 3-19, 1988.

[13] T. F. Fwa and S. A. Tan, "Experimental evaluation of a laboratory twin-probe nuclear gage for specimen density measurement," Journal of Testing and Evaluation, vol. 20, no. 1, pp. 59-65, 1992.

[14] ASTM, "Standard D 2922-05: Standard Test Methods for Density of Soil and Soil-Aggregate in Place by Nuclear Methods (Shallow Depth)," Tech. Rep., ASTM International, West Conshohocken, Pa, USA, 2005.

[15] T. Lunne, P. K. Robertson, and J. J. M. Powell, Cone Penetration Testing in Geotechnical Practice, Blackie Academic \& Professional, London, UK, 1997.

[16] S.-L. Shen, J.-P. Wang, H.-N. Wu, Y.-S. Xu, G.-L. Ye, and Z.-Y. Yin, "Evaluation of hydraulic conductivity for both marine and deltaic deposits based on piezocone testing," Ocean Engineering, vol. 110, pp. 174-182, 2015.

[17] J. L. Ledoux, J. Menard, and P. Soulard, "The penetrogammadensimeter," in Proceedings of the 2nd European Symposium on Penetration Testing, vol. 2, pp. 679-682, Balkema, Amsterdam, Netherlands, 1982.

[18] T. Shibata, M. Mimura, and A. K. Shrivastava, "Use of RI cone penetrometer data in foundation engineering," in Proceedings of the 13th International Conference on Soil Mechanics and Foundation Engineering, vol. 1, pp. 147-150, New Delhi, India, 1994.

[19] M. Mimura, A. K. Shrivastava, T. Shibata, and M. Nobuyama, "Performance of RI cone penetrometers in sand deposits," in Proceedings of the International Symposium on Cone Penetration Testing, CPT'95, vol. 2, pp. 55-60, Linkoping, Sweden, 1995.

[20] M. Mimura and A. K. Shrivastava, "RI-cone penetrometers experience in naturally and artificially deposited sand," in Proceedings of the First International Conference on Site Characterization, vol. 1, pp. 575-580, Atlanta, Ga, USA, 1998.

[21] G. R. Dasari, M. Karthikeyan, T.-S. Tan, M. Mimura, and K.-K. Phoon, "In situ evaluation of radioisotope cone penetrometers in clays," Geotechnical Testing Journal, vol. 29, no. 1, pp. 45-53, 2006.

[22] T. Umezaki, T. Kawamura, and M. Yoshimura, "Investigation of sediment environments in closed water body using RI-density Log," in Proceedings of the 17th International Conference on Soil Mechanics and Geotechnical Engineering, pp. 1050-1053, Alexandria, Egypt, 2009.

[23] R. Jia, T. Hino, T. Hamada, J. Chai, and M. Yoshimura, "Density and undrained shear strength of bed sediment from ND-CPT," Ocean Dynamics, vol. 63, no. 5, pp. 507-517, 2013.

[24] R. Jia, T. Hino, J. Chai, T. Hamada, and M. Yoshimura, "Interpretation of density profile of seabed sediment from nuclear density cone penetration test results," Soils and Foundations, vol. 53, no. 5, pp. 671-679, 2013.

[25] G. Schlieper, "Principles of gamma ray densitometry," Metal Powder Report, vol. 55, no. 12, pp. 20-23, 2000.

[26] M. Karthikeyan, Application of radioisotope cone penetrometer to characterize a lumpy fill [Dissertation, thesis], National University of Singapore, 2005.

[27] W. R. Parker, G. C. Sills, and R. E. A. Paske, "In situ nuclear density measurement in dredging practice and control," in Proceedings of the First International Symposium on Dredging Technology, pp. 25-42, England, UK, University of Kent, 1975. 
[28] K. Preiss, "Non-destructive laboratory measurement of marine sediment density in a core barrel using gamma radiation," DeepSea Research, vol. 15, pp. 401-407, 1968.

[29] M. Karthikeyan and T. T. Soon, "Profiling of heterogeneous soil using nuclear-density cone penetrometer," Geotechnical Testing Journal, vol. 31, no. 6, pp. 513-525, 2008. 


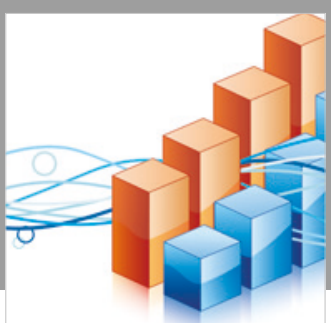

Advances in

Operations Research

\section{-n-m}
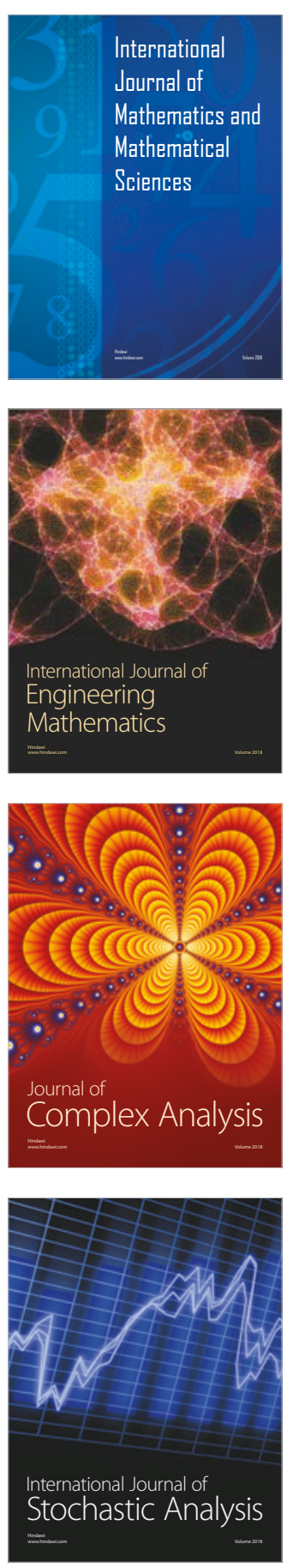
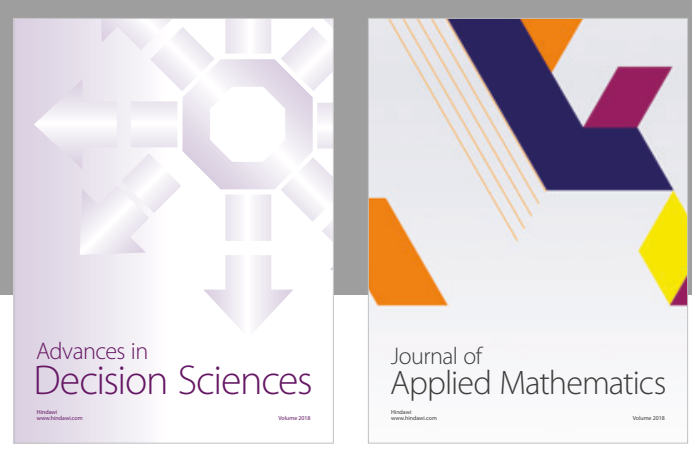

Journal of

Applied Mathematics
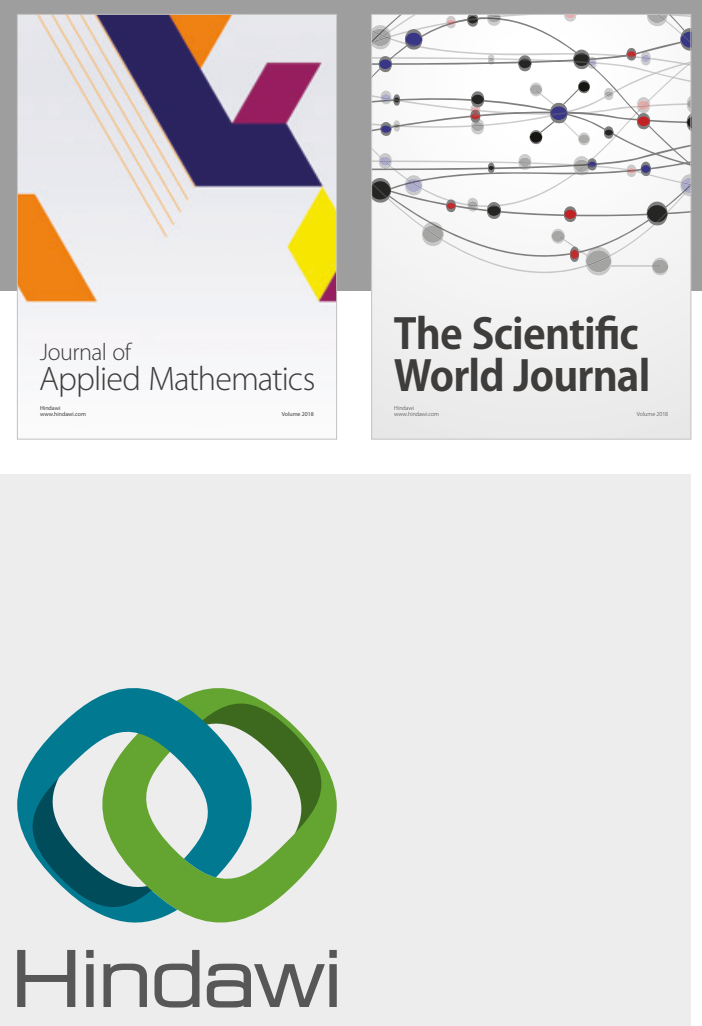

Submit your manuscripts at

www.hindawi.com

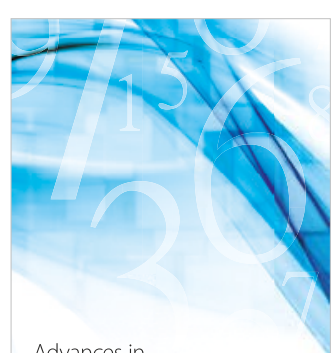

Advances in
Numerical Analysis
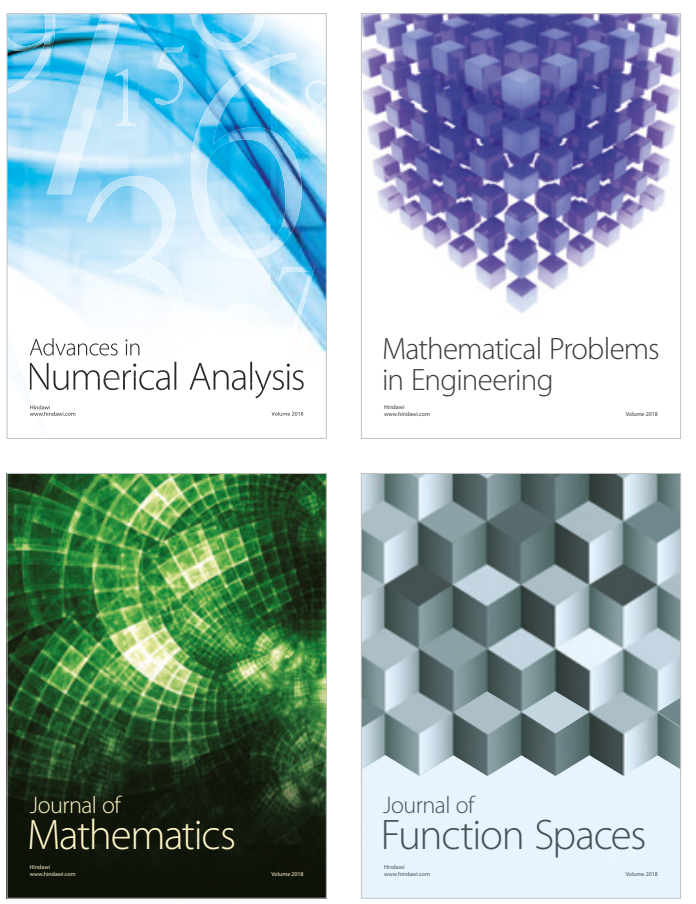

Mathematical Problems in Engineering

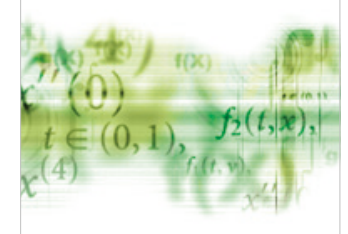

International Journal of

Differential Equations

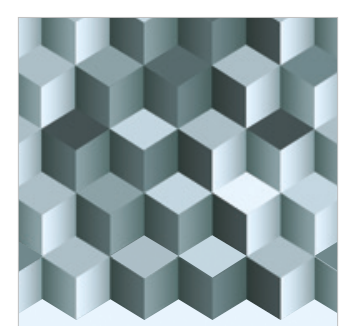

Journal of

Function Spaces

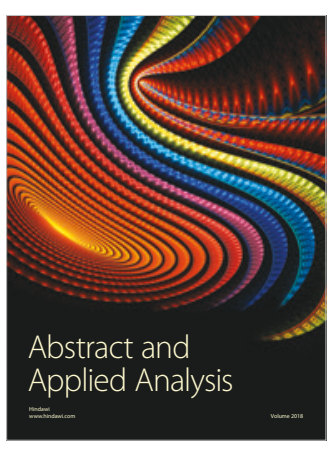

The Scientific

World Journal

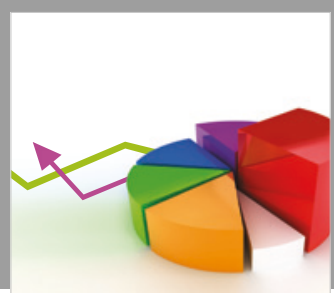

Journal of

Probability and Statistics
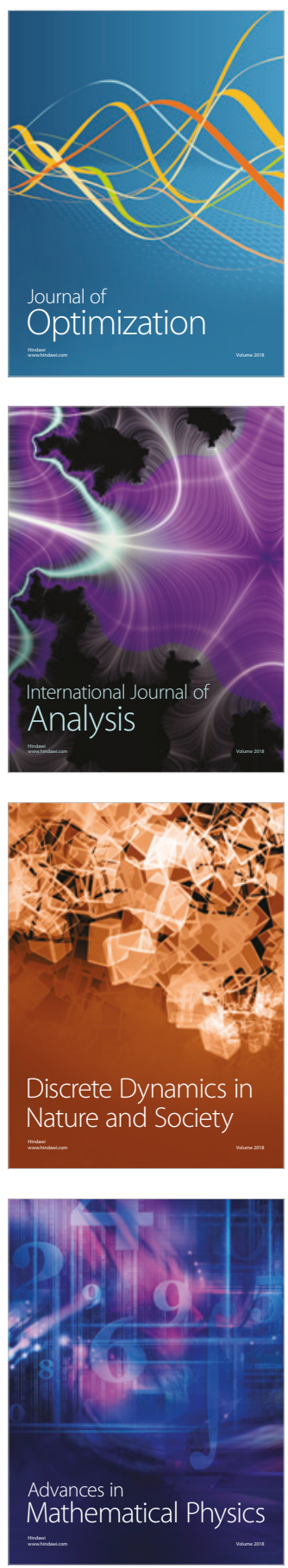